\title{
Entrelacs
}

Cinéma et audiovisuel

Hors-série $n^{\circ} 4$ | 2016

Paysages en séries

\section{Barrages dans la montagne... : le paysage des Morts dans Les Revenants}

BARRAGES DANS LA MONTAGNE... :

Isabelle Rachel Casta

\section{(2) OpenEdition}

Journals

Édition électronique

URL : http://journals.openedition.org/entrelacs/2160

DOI : $10.4000 /$ entrelacs. 2160

ISBN : $2261-5482$

ISSN : 2261-5482

Éditeur

Éditions Téraèdre

Édition imprimée

Date de publication : 1 novembre 2016

ISSN : 1266-7188

\section{Référence électronique}

Isabelle Rachel Casta, «Barrages dans la montagne... : le paysage des Morts dans Les Revenants »,

Entrelacs [En ligne], Hors-série n 4 | 2016, mis en ligne le 06 février 2018, consulté le 22 avril 2019.

URL : http://journals.openedition.org/entrelacs/2160 ; DOI : 10.4000/entrelacs.2160

Ce document a été généré automatiquement le 22 avril 2019.

Tous droits réservés 


\section{Barrages dans la montagne... : le paysage des Morts dans Les Revenants}

BARRAGES DANS LA MONTAGNE... :

Isabelle Rachel Casta

1 «La montagne et la nature ne se contentent pas d'être là, mais dramatisent et imposent à l'histoire une signification. Ce huis-clos monumental et géographique donne l'impression que la seule issue possible, c'est le récit ${ }^{1}{ }^{1}$.

2 "Ogres et princesses perdues ne sont jamais loin dans la forêt automnale des Revenants " (Isabelle Poitte, RLIP).

3 La série fantastique de Fabrice Gobert Les Revenants (Canal+, 2012-2015) accompagne les sombres destins des habitants d'une petite ville de Haute-Savoie coincée entre montagne et barrages, isolat moderne et glaçant où tourbillonnent les vieux papiers d'une catastrophe permanente... Notons tout de suite que les Américains, séduits par l'original ${ }^{2}$ mais peu enclins à suivre une version doublée, ont tourné leur propre version, The Returned $^{3}$ (A\&E, 2015), sous la direction de Carlton Cuse (Bates Motel), en copiant-collant les épisodes et les personnages, et en trouvant près de Seattle le même type de décor - froid, figé et montagneux mais/et sans aucun pittoresque - que le cadre fourni par la région d'Annecy ${ }^{4}$ aux «Revenants » français. Mais le charme n'a pas agi... et le show a été rapidement annulé.

4 Nous nous permettrons de renvoyer à quelques excellents articles critiques: celui de Clélia Cohen "Les Revenants: suite et défunts ${ }^{5}$ " - que nous coderons LRCC - "Le Monument aux Morts ${ }^{6} »$, de Isabelle Poitte (MAM) et « Ils reviennent de $\operatorname{loin}^{7}$ » de Isabelle Poitte encore (RLIP). On peut bien entendu aussi lire « Zombie or not zombie $?^{8}$ » de Paul Sigognac (assez caustique, ZPG), «Hantés par Les Revenants ${ }^{9}$ » de Elvire Emptaz, ou le très sérieux "Les Revenants, Autopsie d'un succès paranormal ${ }^{10}$ ", article d'Eric Mandel dans Le Journal du Dimanche (SPEM). Enfin, strictement pour ce qui concerne le générique, une 
réflexion de Benoit Blanc, «Ces séries qui se font entendre ${ }^{11}$ » (SFE) analyse l'apport du groupe Mogwai à l'atmosphère générale des épisodes.

Rendre crédible l'arrivée des morts dans notre modernité suburbaine est délicat : lumière froide et bleutée, cadrage bizarroïde, limpidité trompeuse d'un lac de retenue... tout est présent pour nous égarer, en juxtaposant au "connu» d'une région française assez banale «l'inconnu » d'un parcours de cauchemar: de l'autre côté des eaux répandues du barrage, vivent les ressuscités... dans une perpétuelle aube livide, qui baigne les "maisons-témoins " adossées à de la végétation sans charme ni couleur. La circulation silencieuse se fait en radeau, où les êtres debout ont l'air en effet de glisser magiquement sur le miroir des eaux... (" un radeau s'éloignant doucement de la rive au clair de lune », rappelle Clélia Cohen, $L R C C$, saison Deux, épisode Un). Le caractère labyrinthique des allées de ces zones pavillonnaires frappées de déshérences est durement éprouvé par Claire Séguret, la « vivante » qui a accompagné sa fille Camille et qui maintenant se croit en butte aux menaces des autres morts.

Pourtant, de grotte inquiétante en chalet perché, de routes hantées - on ne sort jamais de leur circularité - en sentier abrupt, se configure un " paysage de fantaisie » semi-onirique où glissent des silhouettes qui ne devraient pas se trouver là. C'est la collusion du familier et de l'étrange, du coutumier et de l'impossibilium, que synthétise le décor filmé par Gobert, son co-réalisateur Frédéric Goupil et Patrick Blossier ${ }^{12}$; on s'y sent mal, car ces chemins qui ne mènent nulle part (tout est consommé) finissent exactement par évoquer ce qu'ils sont : l'enfer. Un enfer chatoyant en de brefs instants : éclat saphir d'un lac au fond duquel patiente on ne sait quel nocher funèbre ${ }^{13}$... limpidité des eaux de retenue qui ont couvert de leur flot un village englouti.

7 Cette symphonie des bleus et des noirs, des beiges et des gris nous oblige à accommoder un regard sans cesse sollicité entre horizontalité trompeuse des eaux (elles montent, descendent, on s'y plonge aussi pour mourir ou pour ressusciter...) et verticalité anxiogène des montagnes, écrin vite transformé en prison, l'ensemble formant de ce paysage « celui qui inquiète le voir » (selon les propos du philosophe historien de l'art G. Didi-Hubermann). Entre Gregory Crewdson ${ }^{14}$ et Bill Viola ${ }^{15}$, s'ordonne sous nos yeux la scénographie naturalisée d'une sauvagerie enfouie, crevant en de brefs éclairs l'accoutumance géographique qui est la nôtre. A ce propos, l'article Le Monument aux Morts donne longuement la parole à Fabrice Gobert et son équipe (Emmanuel Carrère et Fabien Adda) dont il est le showrunner : ses références sont innombrables et éclectiques, comme en témoigne le court passage suivant :

(...) De la Bible au cinéma de Romero, en passant par la mythologie grecque et Shining (...). Les photos de Gregory Crewdson (...) nous ont accompagnés tout au long du projet, comme le film de Tomas Alfredson, Morse (...). Brett Easton Ellis a également été d'un apport précieux (p. 38).

C'est pourtant dans des références non-dites que se trouvent sans doute les plus fortes réactualisations, ou re-thématisations : Le Village des Damnés (RU, Wolf Rilla, 1960), ou Tour d'écrou...; Victor (qui en fait s'appelle Louis, apparemment tué en 1977 par des "cambrioleurs»), le jeune télépathe au geste omineux (particulièrement réussi et inquiétant) est à la fois très "garçonnet" au sens des années 1970, et pourtant particulièrement offensif : il tue - enfin, pousse au suicide - Mlle Payet, la voisine aux chats, parce qu'elle menace de dénoncer Julie, sa « mère » adoptive, qui pour lui est « la » fée dont lui parlait toujours sa vraie ${ }^{16}$ mère au moment de dire bonsoir. 
10 En effet les procédés de réalisation « donnent vie à la mort » par le filmage singulier de lieux désaffectés, afin de faire percevoir l'essentiel message de l'œuvre, à savoir : qu' « au choc inouï des retrouvailles » il convient de faire se « succéd[er] des abîmes de douleur » ( $M A M)$; on repère tout de suite que le terme d'abîme est au cœur d'un nœud sémantique puisqu'il exprime à la fois le trou vertigineux où l'on tombe, et le procédé esthétique de spécularité. Trois moments permettront de mieux cerner ces imbrications: nous interrogerons d'abord l'intimité du rapport entre le geste du filmage et le surgissement des spectres, puis nous écouterons ces « voix du passé » venues au fil d'une eau de mort et de deuil, pour enfin suivre, au fond de sentes inhospitalières, le lent reflux de ceux dont plus personne ne souhaite la présence.

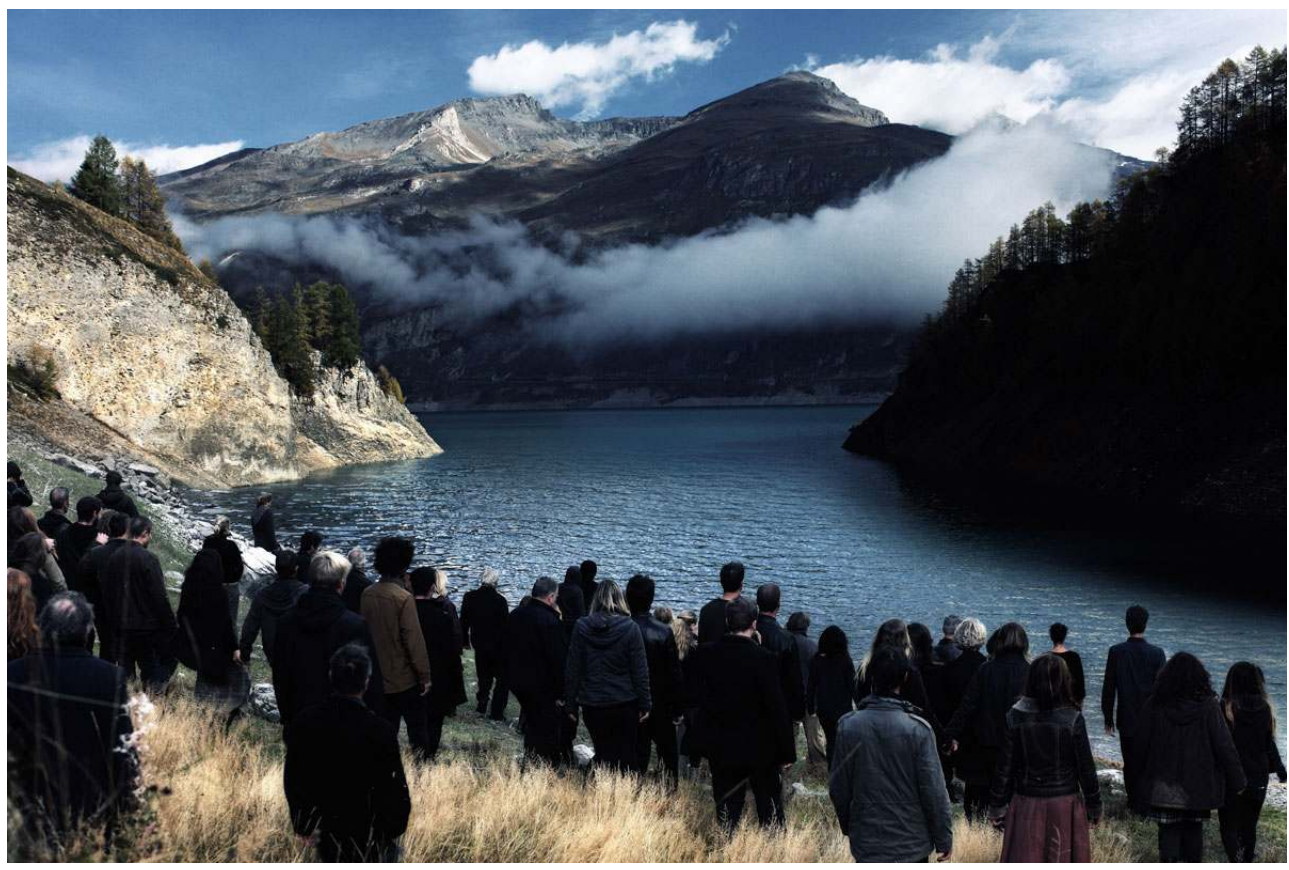

Fig. 1 : Les Revenants

\section{Corps et décors : excéder le cadre?}

En liminaire, qu'il soit permis de rappeler combien le cinéma et les fantômes ont une histoire commune, un engramme (dirait Gilbert Durand), car comme le signale Thomas Schlesser, «à la faculté de capture s'associe celle de répétition. La projection toujours recommencée de ce qui a été fixé au moment du montage ${ }^{17}$ ".

Mais Les Revenants de Campillo et de Gobert n'ont rien de "macabre ", ou même de «tragique » - en tout cas au début; il y a donc une rupture "douce » avec les imagiers précédents, qu'il s'agisse des morts-vivants terrifiants, de Romero à Darabont, ou des apparitions sublimées de Ghost ou de The Ghost and Mrs. Muir ; en effet la série dont nous parlons, inspirée d'un film ${ }^{18}$ lui-même très exceptionnel dans le paysage cinématographique français, est venue bouleverser ces préjugés et redistribuer la donne : sur un schéma directeur im-pensable (au sens strict du terme) Fabrice Gobert (auteur de Simon Werner a disparu) a donc écrit ses «Revenants » avec toute la conviction d'un Georges Franju ${ }^{19}$ ou d'un Will Self ${ }^{20}$, jouant sur les codes de l'étrange plus que du fantastique, ne résolvant rien à la fin (cliffhanger oblige), mais permettant à la mélancolie et à la pensivité du spectateur de se déployer avec une grâce encore inconnue. 


\section{Une expansion contrainte} de Twin Peaks autant que des 4400, suscita un engouement que peu de séries françaises ont réussi à éveiller ces dernières années : la critique aussi bien "savante " que "profane " fut dithyrambique, et le propos suscita controverses passionnées et supputations sans fin : Fabrice Gobert ${ }^{21}$ répondit à de multiples interviews, sans pour autant déflorer le but ou la raison de ces étranges « retours » (la saison deux - automne 2014 - suscita plus de perplexité et - disons-le - un peu moins d'intérêt).

Des morts reviennent en effet, sans malice aucune, dans le petit bourg de montagne où ils ont vécu, quelques mois, années ou décennies auparavant. Et nous, qu'en ferions-nous, de ces morts tendrement chéris mais peu à peu relégués, dépassés, oubliés ? Dans MAM, Isabelle Poitte s'entretient avec le réalisateur afin de cerner l'extraordinaire «filon » que cette idée au fond toute simple - les morts rentrent à la maison - génère et subsume : épinglé sous une vitre, qui se met à battre des ailes... En quelques signes infimes, Les Revenants imprime sa tonalité singulière, entre effroi et poésie. Pas de hordes de zombies ou de fantômes en quête de salut (...) Ce fan de Six Feet Under a su (...) composer un univers ténébreux et étrange, dont le pouvoir d'émerveillement pourrait rivaliser avec les meilleures productions américaines ( MAM, 36).

16 Tout se passe comme si les contraintes occasionnées par le cadre (un barrage, c'est ce qui «barre »! Mais aussi les montagnes escarpées, l'eau menaçante...) s'exprimaient toujours sur le mode de l'outre-passement, du débordement, de la fuite et du trop-plein : la mort se déverse dans la vie, comme les nuages à l'incipit du roman éponyme («(...) onze mois auparavant, de la brume s'était lentement levée dans la vallée et en avait recouvert la surface. Le nuage avait enjambé le barrage avant de se déverser en contre-bas, tel le fantôme d'une chute d'eau prenant la direction de la ville ", p. 9) et comme l'eau de la fin (« l'eau semblait simplement franchir le barrage comme s'il n'existait pas (...). Puis Anton vit le mur d'eau noire se dresser devant lui. Glacée et vengeresse, comme il se l'était toujours imaginé », p. 408) ; mortifère, glacée, inerte, cette eau contient a contrario toute la culpabilité du premier concepteur, l'ingénieur Etienne Berg qui n'a jamais mesuré la fragilité du dispositif, et ainsi précipité des centaines de gens dans la mort... C'est pourquoi son fils, Richard Berg, arrive en renfort au début de la saison deux pour scruter le barrage, afin d'y déceler les traces et les raisons de ces phénomènes.

«Berg» voulant dire "montagne», le corps du personnage s'incorpore à la fluidité mortelle du récit en opposant sa propre résistance au lac de retenue qui par deux fois submerge les lieux. Du coup, l'insolite et le monstrueux ${ }^{22}$ se glissent peu à peu dans une quotidienneté de plus en plus chancelante, et l'on pense à Twin Peaks, ou à Fringe ${ }^{23}$, les " grands modèles » américains.

18

Un cerf fait par exemple quelques pas hésitants au milieu d'une rue, désormais déserte et pillée ; quelques minutes plus tard, il git, blessé à mort, au milieu de la même rue anodine qu'il trempe de son sang, sans qu'aucune présence humaine n'entre dans le cadre: l'horreur naît de ce vide, et non de l'agression elle-même. Cependant on devine que les terribles morsures infligées à la bête sont le fait du fou Serge, et non d'un autre animal, comme semblent le suggérer les habitants au début. Ce genre de détail rappelle l'une des 
autres sources visuelles d'inspiration des réalisateurs : Gustave Doré, le graveur, dont les bruns profonds et les ors éteints trament un décor au sfumato tragique.

\section{Une poétique du barrage}

19 L'argument, rappelons-le, est le suivant: dans une vallée française cernée par les montagnes et proche d'un barrage ${ }^{24}$, des familles bouleversées voient revenir leurs « chers disparus » - tous morts de mort violente d'ailleurs - qui ne savent pas qu'ils sont morts (en tout cas au début) et qui veulent reprendre leur vie là où elle s'est arrêtée il y a deux, cinq, dix ou trente ans. Soulignons tout de suite que cette disposition spatiale contrainte s'adosse évidemment aux souvenirs de la catastrophe de Malpasset, en amont de Fréjus (1959), qui fit 421 morts ; ou, en 1895, celle de Bouzey, dans les Vosges, 100 morts; ou, plus près de nous encore, en Italie, celle de Longarone-Vajon (1963) : 2000 morts (on relève trente accidents de barrage dans le monde entre 1959 et 1987). Lorsque commence la première saison, l'eau se met à baisser sans explication et dégage le clocher du premier village englouti : « au milieu du lac pointait le clocher de l'église en ruines du vieux village. Comme une épine qui tentait de remonter à la surface » (Revenants, p. 217). Chacun des seize épisodes - par le jeu des prénoms, isolés ou duels ${ }^{25}$ - est un memento mori , un tombeau au sens littéraire du terme : mais le dernier de la première saison s'appelle «la Horde» et renoue avec l'imagier des morts-vivants, avançant en meute dans la brume, d'autant plus inquiétants qu'on n'en voit que les traces et les silhouettes ; la petite ville de montagne où se déroulent ces événements ressemble par ailleurs aux "gated communities » américaines : un lotissement, un barrage, des barrières, une étrange police. Une fois l'engloutissement avéré, quelques survivants, quasi-ermites, restent dans les demeures dévastées, comme après chaque grande catastrophe: la Nouvelle-Orléans, Fukushima... L'armée contrôle les lieux, apportant encore une barrière supplémentaire, celle de l'autorité, des interdits sanitaires, de la suspicion généralisée à l'encontre de ces demi-fous qui prétendent rencontrer des morts. Les sous-bois spongieux et détrempés qu'arpentent, désorientés, les « nouveaux morts ", ou encore les forêts inhospitalières où l'on retrouve les corps des gendarmes disparus durant la première saison configurent une nature hostile, très éloignée de la «sylve » originelle des légendes et des robinsonnades, traversée cependant par des routes, des voitures... donc par la technologie humaine.

21 L'importance grandissante de Lucy Clairsene ${ }^{26}$, la jeune "soi-disant » serveuse devenue bergère des morts, permet aux revenants de ne pas rester seuls et désespérés ; ressuscitée elle-même plusieurs fois, elle figure une sorte de ménade, dont la sexualité ouvre les portes de l'apaisement ou de la connaissance; on la sent dépositaire d'un rite ancien, figure d'une « dyonisie » qui rappelle les prostituées sacrées des pratiques initiatiques.

En effet, les huit épisodes de la première saison mêlent achérontique et catabase ${ }^{27}$; le village englouti réactive le sème de la frontière humide, toujours liée à la mort, à la barrière de l'aquaster ${ }^{28}$ qui permet un retour par le biais du miroir des eaux où chacun croise son autre, son double ou son contraire, remonté du fond du temps pour resurgir à la lumière des vivants. Chaque personnage est ainsi le " conducteur " psychopompe d'une entité, mais reste à savoir si les " vivants ", justement, le sont tant que cela.

$\mathrm{Au}$ dernier épisode de la première saison, c'est le contraire qui se produit: les eaux remontent et noient tout ce qui est en contrebas du refuge de Pierre Teissier. Mais le barrage, lui, n'a pas cédé; on se trouve donc devant une aporie : une ville sous les eaux d'un barrage... parfaitement intact ${ }^{29}$. 


\section{Sidération?} contemporain n'est pas toujours bien accueilli : lorsque Michel Costa, le plus âgé des personnages, voit revenir sa femme, Viviane, morte il y a trente-quatre ans, il ne le supporte littéralement pas et se suicide, après avoir mis le feu à sa demeure, mais on ne trouve aucun corps. Cette même Viviane donne au moins trois ou quatre versions de sa mort, disant qu'elle est "morte de faim », ou « dans l'incendie de sa maison », ce qui est démenti par les images, puisqu'on la voit très clairement, juste après la rupture du barrage, aller et venir, fumer, discuter... En fait et enfin, on assiste à sa seule mort: poursuivant son chien qui courait imprudemment sur la plaine enneigée, près de leur chalet, elle disparait en une seconde dans un trou de glace, «avalée » par le décor. Une fois revenue, elle se jette littéralement sur la nourriture, en personnage le plus terre-àterre, le plus "brut de décoffrage" qui soit; croyant toujours que c'est l'heure du « plateau-repas ». C'est une pythie déconcertante, car hyper-matérialiste, mais chargée de rappeler aussi la « vengeance » des victimes du barrage. Sa réjouissante trivialité est le rayon de soleil de cet univers glauque.

A la fin du dernier épisode de la première saison, au petit matin, quand les morts sont repartis, le territoire est de nouveau réduit à la portion congrue puisque tout ce qui ne s'est pas réfugié à " La main tendue », est encore une fois noyé sous les eaux. Est-ce à dire que là résidait entièrement le message des voix du passé, et que les Revenants n'auraient eu qu'une fonction de signes annonciateurs? Peut-être est-ce pour cela que les corps entrent dans le champ comme des apparitions, apportant par les luxuriantes chevelures rousses des femmes ou le rouge éclatant d'un vêtement la stridence soudaine de la vie et de l'animation avant que la palette froide (béton gris du barrage, ocre des chemins, pénombre brune des sous-bois) ne les avale et ne les neutralise de nouveau.

\section{À l'écoute des voix du passé : décor sonore et barque funèbre}

La première manifestation auditive et visuelle d'une série, c'est souvent son générique. Il est ici très programmatique, plastiquement superbe, se déroulant au son d'une "musiquette" faussement candide et "enfantine», mais secrètement chargée d'angoisse, de nostalgie et peut-être de menace... Dus au groupe écossais Mogwai, les soulignements musicaux qui vont napper de leur intensité les moments forts suggèrent une réminiscence assez fidèle du leitmotiv obsédant d'Angelo Badalamenti dans Twin Peaks

Entrelacs, Hors-série $n^{\circ} 4$ | 2016 
, ainsi que des bourdonnements et grincements acousmatiques qui signalent les climax de la série de Lynch. Benoit Blanc parle d'une «intrigante comptine enfantine jouée par quelques notes au xylophone (...) le groupe de Glasgow a su employer des instruments à cordes pour distiller un climat irréel " (SFE). Or on apprend en saison deux que les Revenants que personne n'attend plus restent mutiques - littéralement sans voix - et qu'ils attendent désormais l'enfant de Simon et d'Adèle comme le messie, un messie postapocalyptique, gage de leur retour dans une terre promise qui reste à trouver.

\section{Parole proliférante ou résistante}

Si le couple Adèle/Simon excipe du mythe (inversé) d'Orphée et d'Eurydice - donc lié au chant, au charme de l'incantation - c'est surtout autour de la "voix» de Victor (silencieux en première saison, mais plutôt bavard en saison deux) que se déterminent bien des événements; rappelons qu'il fut "assassiné » il y a trente-cinq ans (1977) par Pierre et son complice (on le sait en entendant ce dernier prononcer exactement la même phrase que celle dite par le tueur qui l'avait débusqué dans un placard) mais il est « revenu » immédiatement après, et a vécu trente-cinq ans près de son père, qui a accepté le « miracle » après un moment d'effarement. Seulement ce dernier, bien sûr, vieillit, et Victor, voyant son " père » mourir d'une crise cardiaque, pousse intérieurement un tel hurlement - «reviens, reviens!» - que tous les morts se réveillent et commencent à souhaiter rentrer.

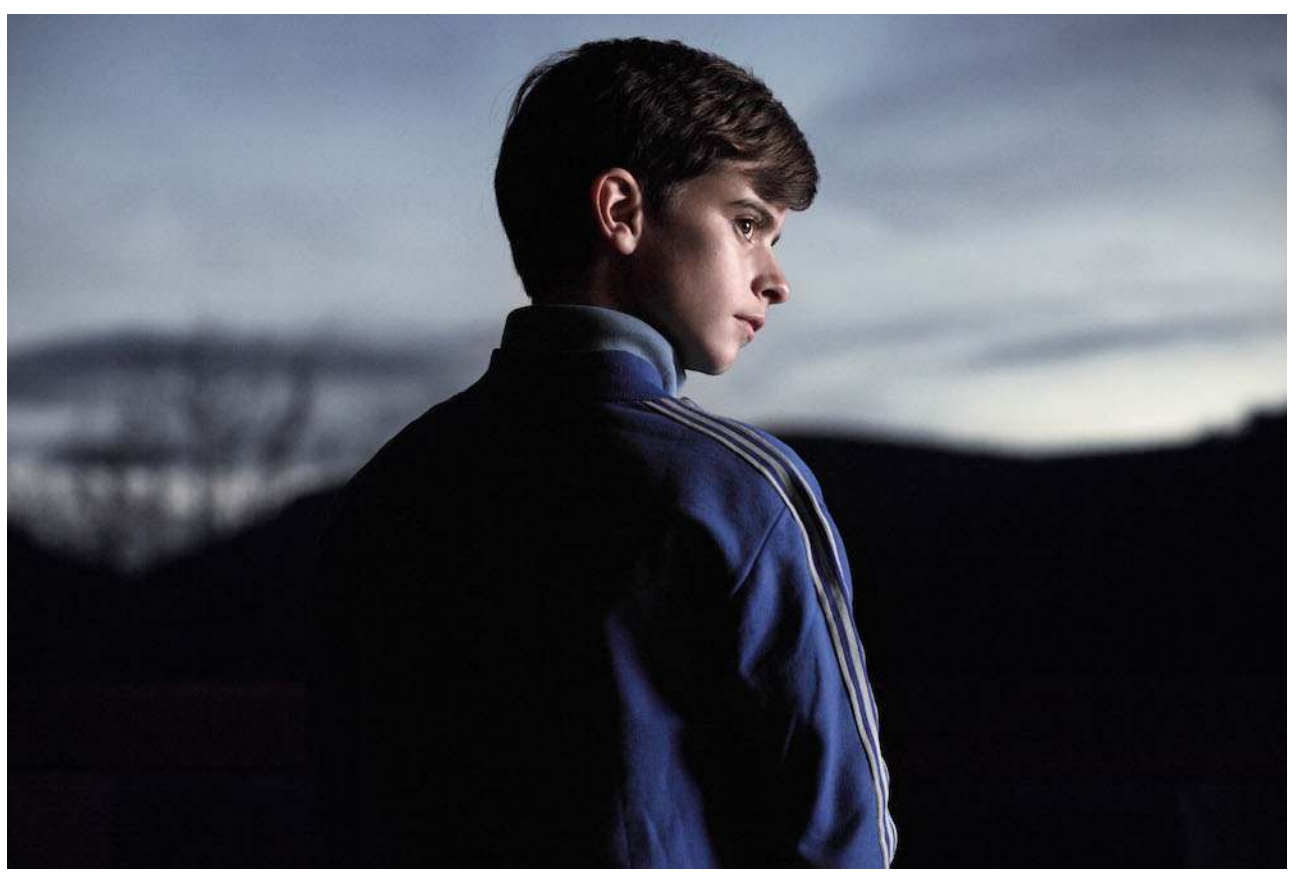

Fig 2: Victor-Louis des Revenants

On peut dire que ce cri d'amour traverse les mondes et ébranle l'au-delà; lui que personne ne croyait jamais (il avait annoncé la chute du barrage, mais Berg n'avait pas écouté) trouve enfin un rôle à sa mesure; il dispute au néant les êtres qu'il aime plus que tout (son père, sauvé mais plongé dans le coma depuis ; Julie, qu'il arrache in extremis au suicide). Alors, Nathan serait-il un deuxième Victor? 


\section{Le cadre dans le cadre}

30 Une essentielle trouvaille visuelle est le constant rappel de l'acte scopique : le «cadre ». Tout évoque en fait le medium (l'écran, borné et focalisateur) au service de la diégèse : tableau, poster, photographies, vitrine à papillon... tout joue sur la mise en abyme, par l'oblicité d'un regard - le nôtre - porté quelques instants sur des fragments d'une autre histoire: l'Exorciste, ou The Ghost and Mrs. Muir... (1947), autrement dit les "grands ancêtres ", modèles/ jalons, qu'il faut dans le même geste homologuer et dépasser. Le fait que le gendarme épie sa femme sur un moniteur de caméra redouble la posture du metteur en scène, scrutant au combo les scènes filmées. Filmage d'un filmage, capture et surveillance s'articulent pour inclure la régie-même des conditions de tournage dans les dispositifs de la fiction. Mais l'exemple le plus marquant est la fenêtre de la maison de Toni et Serge, où se massent, silencieuses et accusatrices, les jeunes femmes assassinées, strictement bornées par les croisillons des carreaux. Leur pâleur spectrale et leurs chevelures ondées renvoient à la dernière image du film d'Amenábar, Les Autres, où s'effacent doucement les visages meurtris de Nicole Kidman et de ses deux enfants.

31 La réclusion dans le deuil pathologique se signale aussi par la circularité piègeante et perpétuelle pour celles qui veulent s'enfuir : la coloration zombifique du dernier épisode (première saison) commence en effet à déteindre sur l'ensemble des comportements et des lieux : tunnel, route et barrage enferment littéralement les fugitifs (Laure, Julie et Victor) dans un dispositif tournoyant particulièrement anxiogène, comme les géographies circulaires des romans fantastiques de Borgès. On ne va nulle part ailleurs que là où on était déjà (c'est la définition même de la névrose); la faille spatio-temporelle commence à se refermer en isolant les témoins de toute possibilité d'évasion. Le mal doit être contenu, contingenté, et l'arche de "La main tendue», pour sectaire et apocalyptique qu'elle soit, figure encore le seul recours des habitants.

C'est auprès d'une piscine pleine de feuilles mortes, métaphore puissamment mélancolique au cœur du Domaine abandonné, balayé par le vent automnal et surplombé par le gris-vert minéral de la roche, que Camille devra apprendre à ses « amis » Audrey et Esteban qu'ils sont morts. Encore un cadre, encore de l'eau morte, miroir glauque qui ne reflète plus que le ciel tourmenté.

\section{Visages dans la nuit transparente.}

... Et jamais la mort ne l'emportera ${ }^{30}$

Lorsqu'on découvre que Lucy, la jeune serveuse étrange, est « morte », elle aussi, sous les coups de Serge Guillard ${ }^{31}$, le tueur en série - comme Julie l'infirmière, terriblement blessée, sinon tout à fait cliniquement morte - les certitudes basculent encore un peu plus ; chacun se rachète comme il le peut lors de cet intermède salvifique, auquel nul n'aurait pu s'attendre, pour suturer les plaies demeurées ouvertes, et refermer les plus sanglantes blessures.

\section{Procédures et procédés.}

Il en ira ainsi de Léna, sœur de Camille, qui s'échappe de l'hôpital où on la soigne pour l'horrible cicatrice purulente qu'elle a dans le dos; elle est en fait ramassée par Serge, le 
fameux tueur, lui aussi revenu d'entre les morts. Mais cette fois, il ne lui fera aucun mal, préférant passer ses pulsions sadiques sur des animaux devenus substituts totémiques de la jeune fille. Le décor se décentre alors, s'éloignant de la ville fantôme pour remonter les pentes vers la maison de Toni et Serge, que le retour du père en saison deux retransforme en charnier.

Hôpital à demi abandonné où accouche Adèle, néon blafard et faiblissant, grésillement, baisse d'intensité ou clignotement éminemment dramatique de la fiction accompagnent la saison deux, même si leur simple réédition tourne parfois au procédé. Rompant délibérément avec l'apparence «réaliste» du début, la scène dite de la "grotte de l'écorché » précipite Adèle et Simon dans une dimension encore autre, puisqu'elle en ressort vêtue en mariée, prête à convoler spectralement avec son fiancé. Allégorie, fantasme d'une dépressive, suicide déguisé ? On ne sait, mais cette brusque plongée dans les entrailles de la montagne a tout de l'initiation, sinon maçonnique, du moins ésotérique. Ce qui peut amener à une autre forme de remarque : les prénoms dessinent eux aussi un réseau subtil d'allusions et d'allégeance: "Morgane » et "Viviane » en appellent aux merveilleux arthurien, Virgil nous guide, comme autant de nouveaux Dante, au cœur des chemins d'un enfer revisité, Lucie et Claire (Séguret) «éclairent » et Esther et Nathan, fils de Simon, évoquent la tradition biblique et apostolique, surtout flanqués de Pierre (Teissier), de Paul («frère » de Louis) et de Thomas.

A l'image augurale de la saison deux (une ambulance fonce, solitaire et tous feux allumés, sur la route obscure du barrage qui finit en impasse) viennent se greffer une infinité de plans, quasi immobiles, de silhouettes à peine détachées des fonds ombreux où le cadre les invite: pendant ce temps, les appartements sont envahis de mouches, pourriture, cancrelat, infestation silencieuse des signes de corruption, dans le décor et le corps. Les bondes des lavabos laissent passer d'infects liquides de décomposition, comme si le décor était chargé d'exsuder et de matérialiser l'état abandonnique et frelaté des relations humaines, où le croupissement des affects empoisonne toute relation possible.

\section{Pourrissements et blessures}

En effet la parousie ne dure pas: escarres, plaies et ecchymoses "signalent» que la corporéité des défunts est fragile, menacée et, bien entendu, provisoire. Le visage de Camille commence à se défaire, malgré les efforts de sa mère et de sa soeur pour maquiller les traces de décomposition. Victor porte au bras une plaie qui s'étend. Serge demeure dans son souterrain, de plus en plus couvert d'escarres, comme pour expier enfin ses crimes. On se souvient encore de ce que Godard, dans Alphaville (1965), faisait dire à la machine et à Lemmy Caution: "Quel est le privilège des morts ? - Ne plus mourir » : Gobert va nous prouver le contraire. L'électricité coupée, les magasins pillés et ouverts à tout vent, les déchets tourbillonnant dans les rues vides manifestent bien, en effet, l'arrivée de l'Ange de la Mort : Lucy, la serveuse en nocher des Enfers court vêtu, mais cependant redoutable.

Ces innombrables moments où les corps altérés ou modifiés forment un nouveau baroque, où l'horrifique et l'émouvant s'épousent sans contrainte, écrivent une esthétique du " comble ", qui s'actualise ici dans le choix du jeune fantôme Victor/Louis Levanski ${ }^{32}$, comme «icône »; c'est son regard noir, ses traits figés indéfinissablement inquiétants et sa pâleur déconcertante qui «marquent » le best-seller inspiré par la série. Son visage et son corps deviennent à leur tour décor, à force d'être scrutés et surinvestis d'affects et de 
présage, illustrant le vers du poète gallois : « After the first death, there is no other. » (Dylan Thomas) surface ", en saison Deux "Le phénomène se répand», au versus de celle du film: "l'impensable s'est produit »), mais ne passe pas pour autant, car il confronte des temps, des affects et des moments de l'être trop différents : avoir quinze ans quand sa jumelle en a maintenant dix-neuf, rencontrer pour la première fois sa fille de dix ans quand on en a soi-même vingt-trois pour l'éternité ou encore revoir face à face le frère qu'on a abattu parce qu'il avait poignardé des femmes à mort n'est évidemment pas soutenable longtemps. Au fond, tout se passe un peu comme si au « ou » du choix déchirant et de la privation insupportable mais inéluctable, les revenants avaient voulu substituer, un bref moment, le « et » de la coexistence des contraires, de la fin et du recommencement, de l'exil et du royaume où vivent et meurent les Hommes...

Poitte aura le provisoire dernier mot : «Face à leur proche revenu des limbes, les vivants semblent évoluer au bord de l'abîme» (MAM).

\section{Filmographie}

Les Revenants, Robin Campillo, 2004.

The Others (Les Autres), Alejandro Amenàbar, 2001.

The Sixth Sense (Le Sixième sens), M. Night Shyamalan, 1999.

Sous le sable, François Ozon, 2000.

Ghost, Jerry Zucker, 1990.

Truely, Madly, Deeply, Anthony Minghella, 1991.

Vers l'autre rive, Kiyoshi Kurosawa, 2015.

La fille et le fleuve, Aurélia Georges, 2015.

Ni le Ciel ni la terre, Clément Cogitore, 2015.

Sériographie

Les Revenants, Canal+, 2012-2015.

The Strain, FX, 2014-présent.

Entrelacs, Hors-série $n^{\circ} 4$ | 2016 
The Walking Dead, AMC, 2010-présent.

In the Flesh, BBC Three, 2013-2014.

Six Feet Under, HBO, 2001-2005.

Twin Peaks, ABC, Showtime, 1990-1991.

Bibliographie

Les Revenants, Laura Kasischke, 1999.

Les Revenants, Seth Patrick, trad. Sébastien Baert, 2014.

\section{NOTES}

1. Caroline Benjo, productrice, citée par article LRCC, p. 29.

2. Plébiscitée par la critique anglo-saxonne, la série a reçu un International Emmy Award en 2013 (Hollywood).

3. Une seule saison, car annulée au dixième épisode pour cause de faible audience. Visible sur Netflix.

4. Mais ce sont les ruines du barrage de Malpasset qui fournit le décor le plus impressionnant des scènes d'extérieur.

5. Cohen, Clélia. «Les Revenants : suite et défunts ", Libération, 26/27 septembre 2015, pp. 27-29.

6. Poitte, Isabelle. « Le Monument aux Morts », Télérama 3280, 21.11.12, p. 36-40.

7. Poitte, Isabelle. «Ils reviennent de loin », Télérama 3428, 23.09.15, pp. 75-77.

8. Sigognac, Paul. «Zombie or not zombie?», ELLE, 28.09.15. Repéré à http://www.elle.fr/ Loisirs/Series/Les-Revenants-notre-avis-sur-la-nouvelle-saison-sans-spoilers-2993267, consulté le 19 juillet 2016.

9. Emptaz, Elvire. « Hantés par Les Revenants », ELLE Culture, 23.11.12, p. 47.

10. Mandel, Éric. "Les Revenants, Autopsie d'un succès paranormal ", Le Journal du Dimanche, 20.09.2015. Repéré à http://www.lejdd.fr/Medias/Television/La-serie-Les-Revenants-autopsie-dun-succes-paranormal-751919, consulté le 19 juillet 2016.

11. Blanc, Benoit. «Ces séries qui se font entendre ». Libération. 3.10.2015, p. 38.

12. Chef opérateur, n'a travaillé qu'avec une seule caméra car «il est impossible de régler une lumière parfaite pour deux angles de vue différents et simultanés » (LRCC).

13. Dans le roman, l'être entre deux mondes est décrit ainsi : «(...) se tenait une silhouette. Humaine. Crasseuse. La tête baissée, elle se couvrait le visage avec ses mains. Son corps nu était couvert de boue. L'individu baissa les mains et leva la tête. En apercevant son visage, Anton battit en retraite en hurlant. » in Patrick, Seth. Les Revenants, trad. Sébastien Baert. Paris : Éd. Michel Lafon, 2015. pp. 408-409.

14. Photographe américain né en 1961 : ambiance crépusculaire, personnages figés, scène de crime peut-être. «(...) comme dans les photographies de suburbs américaines apparemment anodines de l'artiste Gregory Crewdson, grande référence plastique des Revenants ». LRCC. 
15. Vidéaste américain né en 1951 ; exposé en 2014 au Grand Palais : installations monumentales, corps en suspension («L'ascension de Tristan »), poèmes visuels, il est vrai très proches des effets de figements dans Les Revenants.

16. Laquelle ressuscite en saison II, au grand désespoir de Julie. Mais elle n'est qu'une énième mère adoptive, Victor venant de plus loin encore, tout entier voué à avertir les hommes des catastrophes à venir et régulièrement rejeté et nié.

17. Schlesser, Thomas. "Cinéma", in Di Folco, Philippe. Dictionnaire de la mort. Paris: Éd. Larousse, 2010, pp. 228-231, p. 231. Il rappelle la profonde aversion d'André Bazin pour les morts filmées sans ellipse, procédé dont Bazin soulignait l'obscénité intrinsèque dans Qu'est-ce que le cinéma ? Bazin, André. Qu'est-ce que le cinéma ? 1, Ontologie et langage. Paris : Éditions du cerf, 1958.

18. Les Revenants, Robin Campillo, 2004.

19. Cinéaste français (1912-1987).

20. Ecrivain anglais de nouvelles fantastiques grinçantes, né en 1961 ; a écrit en particulier Ainsi vivent les morts (2000).

21. Assisté d'Emmanuel Carrère et Fabien Adda. Pour la saison 2, Carrère s'en va (mais reste en back-up), et Audrey Fouché arrive ; seule française à avoir participé à l'écriture des "Borgia » sous la férule de Tom Fontana, elle professe une admiration sans borne pour sa «bible ", Écriture: mémoires d'un métier, de Stephen King. Dans le ELLE du 2 octobre 2015, elle précise que faire avancer 15 personnages sans se tromper relève d'un «immense jeu de dominos » (p. 170). Coline Abert participe elle aussi au scénario.

22. L'une des revenantes, Audrey, finit par dévorer le corps de Sandrine, sa mère, morte à côté d'elle (saison II, dernier épisode).

23. Cette série de J.J. Abrams, Alex Kurtzman et Roberto Orci (2008-2013) s'inscrit dans la « fringe science », littéralement les sciences « marginales».

24. En fait, deux: le premier, détruit après la catastrophe, est plus ou moins couvert de végétation ; l'autre, récent, forme la route sur laquelle Laure et Julie tentent de s'enfuir... et d'où Michel Costa va se suicider en se jetant dans le vide.

25. « Camille» (accident de car) ; « Simon » (suicide) ; « Julie » (vivante, mais ancienne victime du tueur en série Serge); "Victor » (assassiné avec ses parents adoptifs en 1977 ; en fait son père a survécu); «Serge et Toni » (Serge, tueur et tué ! Toni, futur suicidé...) ; «Lucy » (sans doute déjà «revenante» et de l'agression de Serge... et de celle de son père, Milan, trente ans avant) ; «Adèle " (a voulu attenter à ses jours après la mort de Simon); «La Horde ». Pour la saison 2 : «L'enfant» (ép. 1 : c'est Nathan, le fils de Simon et Adèle); «Milan » (ép. 2 : père de Serge et Toni, il apparaît comme l'incarnation du mal et du crime); "Morgane» (ép. $3:$ le petit-ami assassiné de Lucy) ; «Virgil » (ép. 4 : jeune mort et futur amoureux de Camille) ; "Mme Costa » (ép. 5 : revenante menteuse et triviale, mais douée d'une quasi sixième sens) ; «Esther » (ép. 6 : encore une jeune morte, tuée par Serge ; elle revient pour le hanter, il lui demande pardon...); «Etienne» (ép. 7 : l'ingénieur responsable de l'effondrement du barrage, 35 ans avant) ; «Les revenants » (ép. 8, ce qui symétrise le dernier chapitre de la première saison, « La Horde »).

26. Clarsen, dans le roman éponyme.

27. La catabase est l'un des rites les plus fréquents de l'épopée grecque : il s'agit de descendre ( « cata ») chez les morts, pour en rapporter un savoir ésotérique ou chamanique... L'achérontique (id est lié à l'Achéron, l'un des fleuves des Enfers) désigne l'ensemble des ouvrages rédigés, pense-t-on, par les Etrusques et traitant des questions de l'au-delà.

28. Ce terme latin, qui désigne l'« ensemble des phénomènes liés à l'élément liquide » est présent dans Les Structures anthropologiques de L'Imaginaire, de Gilbert Durand, afin de montrer les accointances qui unissent l'eau, la féminité et la mort.

29. Les Revenants, p. 412 : «Un à un les autres le rejoignirent et ils contemplèrent la scène. Les immeubles submergés. La ville engloutie ».

30. 25 poèmes, « And death shall have no dominion », Seuil, 1970, p. 413. 
31. Mais l'épisode 3 de la saison Deux, "Morgane ", nous la montre comme se faisant déjà tuer par Milan, juste après l'effondrement du barrage, trente-cinq ans plus tôt...

32. Le jeune interprète, Swann Nambotin, a acquis une renommée foudroyante et immédiate, reléguant dans l'ombre quasiment l'ensemble du casting.

33. En araméen, ce nom signifie «don de Dieu »; la tradition voit en lui un des disciples, peutêtre celui dont il est dit : « le disciple que Jésus aimait »; mais il n'apparait que dans l'évangile de Jean.

34. Garrigos, Raphael et Roberts, Isabelle. "Les Revenantes ", Libération, 30/12/2012. Repéré à http://next.liberation.fr/culture/2012/12/30/les-revenantes_870783, consulté le 19 juin 2016.

\section{AUTEUR}

\section{ISABELLE RACHEL CASTA}

Isabelle-Rachel Casta enseigne la littérature comparée à l'Université d'Artois (Gracq, Jünger, Buzzati); elle est spécialiste des cultures fantastique, noire ou criminelle. Auteure de nombreux articles (" De battre son cœur s'est arrête... : mourir en série dans Buffy the Vampire slayer », Proteus, «Eclairer le discours médico-légal » Implications Philosophiques...), elle rédige en ce moment un ouvrage sur les séries d'autopsies pour les éditions Vendémiaire. 